\title{
Solution-Processable White-Light-Emitting Germanium Nanocrystals
}

\author{
Naoto Shirahata ${ }^{\dagger, \ldots, s, *}$ \\ †nternational Center for Materials Nanoarchitectonics (WPI-MANA), 1-1 Namiki, \\ Tsukuba, Ibaraki 305-0044, Japan \\ National Institute for Materials Science (NIMS), 1-2-1 Sengen, Tsukuba, Ibaraki \\ 305-0047, Japan \\ §Precursory Research for Embryonic Science and Technology (PRESTO), Japan \\ Science and Technology Agency (JST), 4-1-8 Honcho Kawaguchi, Saitama 332-0012, \\ Japan
}

*Corresponding author:

E-mail address: SHIRAHATA.Naoto@nims.go.jp (N. Shirahata)

Keywords: Germanium, Nanocrystals, Oleylamine, White light, Photoluminescence, Column chromatography 
ABSTRACT

This paper describes an efficient chemical route for the synthesis of visible light emitting nanocrystals of germanium (ncGe). The synthesis started by heating $\mathrm{Ge}(\mathrm{II})$ iodide at $300^{\circ} \mathrm{C}$ in argon atmosphere. Spectroscopic characterizations confirmed the formation of diamond cubic lattice structures of ncGe. By grafting hydrophobic chains on the ncGe surface, the dispersions in nonpolar solvents of the ncGe became very stable. The as-synthesized ncGe showed the bluish white photoluminescence (PL) feature, but it was found that the PL spectrum is composed of many different emission spectra. Therefore, the color-tuning of white light emission is demonstrated through the witting removal of extra ncGe with unfavorable emission feature by making full use of column chromatographic techniques. 


\section{Introduction}

White light emitting materials have widely attracted a lot of interests because of their versatile use as the emission sources for displays, illuminations, flashlights, and vehicle headlamps in artificial lighting industry [1]. To date, phosphors containing rare-earth ions have allowed for the broad use in those applications. The emission color depends on the rare-earth ions including europium, cerium and terbium [2-6]. Combining emission colors result in white lights. White light emitters are easily fabricated only by coating the emitting materials on blue-LED diode. Although such light emitters with rare-earth ions show high durability against heat and chemicals, the luminescence intensity tends to saturate rapidly with increase of excitation power due to a long lifetime of mili-second level. Besides, the unstable supply of rare-earth elements accelerates the development of rare-earth alternatives [7]. In order to serve this need, both organic dyes and nanocrystals of semiconductors including silicon are active in the front line of the advanced white-light emitting materials [1, 8-12].

Germanium $(\mathrm{Ge})$ is a typical semiconductor with $0.67 \mathrm{eV}$ of small bandgap, and has a significantly poorer optical performance due to its indirect bandgap character. The discovery of yellow photoluminescence (PL) from nanocrystals of Ge (ncGe) has therefore created an opportunity for its optical functionality, and opened a long-running 
debate on its PL origin [13]. Ge is also one of environmentally friendly and nontoxic elements as well as silicon. Therefore, the industrial use of Ge as a light emitting source is promised to open fascinating area. It is known that the quantum confinement effect appears in ncGe when its physical size is getting smaller than bulk exciton Bohr radius for the crystal $(\leq 11.5 \mathrm{~nm})$, resulting in the discrete of energy gap. Bulk exciton Bohr radius $\left(\alpha_{B}\right)$ can be expressed as:

$$
a_{B}^{*}=\frac{4 \pi \hbar \varepsilon_{0} \varepsilon_{r}}{e^{2} \mu}
$$

where $\mu$ is the reduced mass, $\varepsilon_{\mathrm{r}}$ is dielectric constant and $\hbar$ is reduced Planck constant.

One of the distinctive advantages of Ge over Si for optical applications is indeed the large bulk exciton Bohr radius caused by the smaller effective mass of its electron-hole $(e-h)$ pairs and its larger static dielectric constant compared to silicon. Due to the large exciton Bohr radius, it can be expected that its size reduction further strengthens the $e-h$ carrier confinement compared to Si. Nowadays, it is reported that the emission is tuned in a remarkably wide range in photon energy of $0.83-3.87 \mathrm{eV}\left(\lambda_{\mathrm{em}}=320-1500 \mathrm{~nm}\right)$ [14-24]. Some of the ncGe are known to emit the light efficiently with $10-20 \%$ of PL quantum yields (QYs) $[15,17,19]$; however, there is no commercially available ncGe at present. A possible reason for the unavailability lies in the fact that colloidal chemistry for ncGe synthesis has remained undeveloped. Due to the covalent character of ncGe, it 
is reasonable to require high energy to form the diamond cubic lattice. However, most of colloidal syntheses are carried out in very mild conditions such as a room-temperature synthesis. Among numerous published accounts of colloidal routes, the use of $\mathrm{GeI}_{2}$ as a starting precursor provides the feasible pathway to yield diamond cubic lattice structures of ncGe. Korgel's group reported the colloidal synthesis of ncGe at $300^{\circ} \mathrm{C}$ by taking advantage of the high reactivity of $\mathrm{GeI}_{2}$ and high $\mathrm{GeI}_{2}$ solubility in alkyl phosphines [25]. Chemical reduction of $\mathrm{GeI}_{2}$ with oleylamine was reported by Klimov and co-workers, but the emissions of the products were seen in the near-IR (NIR) region [17]. Similar NIR PL feature was reported in elsewhere [24]. To the best of my knowledge, visible light emission has not yet been observed from ncGe synthesized by chemical reduction of $\mathrm{GeI}_{2}$. Thus, tuning the color of white-light emission is still challenging for ncGe.

The present paper reports first the colloidal synthesis of visible light emitting ncGe using a mixture of $\mathrm{GeI}_{2}$ and oleylamine. Furthermore, tuning the color of white light is demonstrated by control over size distribution of the ncGe.

\section{Experimental}

\subsection{Chemicals}

Oleylamine ( $>97 \%)$ was purchased from Acros Kanto and was used after degassing under 
vacuum. $\mathrm{GeI}_{2}(99.99 \%, \mathrm{Ge})$ was purchased from Strem chemicals. All reaction was carried out under argon atmosphere using a standard Schlenk line technique.

\subsection{Colloidal Synthesis of ncGe}

As a typical experiment, $10 \mathrm{ml}$ of oleylamine was taken in a Schlenk flask and degassed at $100^{\circ} \mathrm{C}$ under vacuum for about $1 \mathrm{~h}$. In another flask, $0.5 \mathrm{mmol} \mathrm{GeI}_{2}$ was taken inside the glove box and also degassed under argon. Oleylamine was then injected into the flask of $\mathrm{GeI}_{2}$ for mixing with a syringe. The mixture was then stirred with heating at $120{ }^{\circ} \mathrm{C}$ to dissolve in argon bubbling. After complete dissolution, the mixture was then heated to $300{ }^{\circ} \mathrm{C}$ under a constant argon flow for 2 hrs. During the reaction, the color of the solution changed into orange brown, indicating the formation of ncGe. The product was purified by adding methanol to the final solution. Next, the solutions were centrifuged with a speed $10000 \mathrm{rpm}$ to separate a dark-brown solid which is ncGe emitting the NIR lights from the product [23]. The NIR luminescent ncGe was easily removed from the product because the solid was precipitated at the bottom of the centrifuge tube. The high boiling temperature of the oleylamine allowed for evaporation of methanol and dispersal of the ncGe within the unreacted oleylamine. The product obtained from supernatant solution emits the bluish-white light when excited with $365 \mathrm{~nm}$ of handheld lamp. This centrifugation was carried out as the first step for purification. A second purification process was performed using a conventional silica gel column chromatography with eluent of 
dichloromethane. In a third purification process, the sample was then subjected to the size exclusion chromatography to achieve the separation of ncGe by emission color.

\subsection{Characterization}

Structural phases of the products were characterized with Raman spectroscopy. Raman spectroscopic measurements were carried out in laser Raman microscope with $785 \mathrm{~nm}$ laser excitation source (Raman II, Nanophoton Corporation). TEM images were obtained by operating a JEOL JEM 2100 electron microscope at $200 \mathrm{kV}$. Size distributions of the samples were measured by dynamic light scattering (DLS, Nikkiso UPA-UT151, Japan). Optical absorbance and emission properties were measured using quartz cuvettes with NCs dissolving in non-luminescent dichloromethane. The optical absorbance was recorded by UV-VIS spectrophotometer (V650, JASCO). Photoluminescence (PL) and PL excitation (PLE) spectra were measured using fluorescence spectrometer (Fluoromax, Horiba Jobin Yvon). Since oleylamine shows a weak blue light emission, the emission was subtracted as a background to

give PL spectra of the samples.

\section{Results and Discussion}

$\mathrm{GeI}_{2}$ is known to disproportionate at $350^{\circ} \mathrm{C}$ to yield $\mathrm{Ge}$ and $\mathrm{GeI}_{4}$ [26], but nanocrystals form at $300^{\circ} \mathrm{C}$ in the present study. At least, there are two possible routes to explain the ncGe synthesis at lower temperature. In the present reaction system for ncGe synthesis, $\mathrm{GeI}_{2}$ reacts 
with oleylamine to form adducts of $\mathrm{GeI}_{2}$ and oleylamine at $120^{\circ} \mathrm{C}$. The adduct formation is confirmed through the observation that $\mathrm{GeI}_{2}$ dissolved completely in oleylamine at the temperature, and gives an opportunity for ncGe synthesis under mild reaction temperature of $300^{\circ} \mathrm{C}$. In the other route, it might be possible that a terminal amine of the starting oleylamine serves as a reducing agent yielding $\mathrm{Ge}$ and $\mathrm{GeI}_{4}$. Instead, oleylamine is oxidized to imine or nitrile during the reductive reaction as evidenced in the infrared study [23]. In this reaction, the resultant molecules with either one of amine, imine and nitrile groups also serve as capping agents to prevent the aggregation and the enormous growth of nanocrystals. Owing to surface modification with the molecules, the products were soluble in any nonpolar solvents.

The orange-brown color solution product was centrifuged in a mixture of toluene and ethanol for 5 min with $10000 \mathrm{rpm}$ of rotation speed. The dark-brown precipitate was composed of NIR light emitting ncGe [23], but the supernatant orange solution contained ncGe emitting the visible lights as described below. The supernatant solution was diluted with dichloromethane for a next step of purification after several cycles of the centrifugation, and was then subjected to conventional silica-gel column chromatography in a second step for purification. The purification process was monitored on a plate of thin layer chromatography (TLC). The purified product showed the bluish-white PL property when excited with $365 \mathrm{~nm}$ of handheld UV lamp. Henceforth, this bluish-white light emitting ncGe was used as a parent sample. 
Crystallographic characterization of the parent sample was carried out using DLS, TEM and Raman. Size distribution was measured by DLS, and presented in Fig. 1(a). Nanocrystals bigger than $12 \mathrm{~nm}$ are no longer contained in the parent sample, because the first purification process with centrifugation allows for the complete removal of NIR-light emitting ncGe with average diameter of $12 \mathrm{~nm}$ [23]. As is clearly seen, the size distribution of ncGe is broad in the region of 1-12 nm, and the peak top of the distribution is seen at around $4.8 \mathrm{~nm}$. Fig. 1(b) shows TEM photograph and selected-area electron diffraction (SAED) pattern of the parent sample. Shapes of the black-color dots were mostly spherical, and the diameters of the dots are almost smaller than $12 \mathrm{~nm}$ consistent with the results obtained by DLS measurement. The SAED pattern of inset covers the TEM observation area. The rings displayed were assigned to the crystal planes. Three diffraction rings matched well with crystal spacing of (111), (220), and (422) planes, corresponding to the diamond cubic lattice structure of Ge. Thus, the SAED pattern, which is performed in conjunction with the TEM observation, revealed that the black dots are diamond cubic lattice structure of ncGe. A typical high-resolution image in Fig. 1(c) resulted that the ncGe is composed of single crystalline phase of diamond cubic lattice. Raman spectrum of the parent sample is presented in Fig. 1(d), and is compared to that of bulk crystalline Ge wafer. In view of UV-VIS spectrum (not shown), this sample should not absorb photon of $785 \mathrm{~nm}$ of incident light for Raman measurement; however, maybe little emission excited with the light 
distorts significantly the Raman spectral shape by pushing up the base line. The sample spectrum shows that the Raman transverse optical (TO) mode is broadened from that of bulk Ge, and has a long tail to the lower frequency due to the decrease in size of ncGe, but its peak position is shifted only a very small amount. Similar trends in Raman spectral line of ncGe have been reported in elsewhere [27].

Although bluish-white light emissions have been commonly reported in many papers, but tuning the color of white light still remains difficult [28-30]. Our previous study used silicon nanoparticles as a model to demonstrate the successful separation of nanoparticles by emission color through conventional silica-gel column chromatography [31]. The use of column chromatographic techniques offers an efficient extraction of the predefined nanoparticles without loss [31-34]. In the present study, the tuning the color of white light was challenged based on the assumption that the bluish white PL spectrum is described as ensemble of different emission lines. To achieve this goal, a high performance liquid chromatography (HPLC) with size exclusion column was used. In a representative case, the parent ncGe sample was injected on to a size exclusion column, which resolved the mixture of NCs into multiple fractions, and the separated fractions were monitored via refractive index (RI) detection, as shown in Fig. 2(a). As marked in the chromatogram, three fractions were extracted from the parent sample. In the HPLC treatment, chloroform was used as an eluent. So, it is reasonable that all of the extracted 
components have positive RI values of HPLC peaks. Two more isolated peaks are seen in the chromatogram based on the RI detection signal, but luminescence signals were not seen with naked-eyes from these fractions. The purpose of this study is the tuning the color of white PL using ncGe. Therefore, these two most non-emissive (or extremely low PL signal species) were not subjected to further study.

The HPLC process allowed for isolation of ncGe with different size distributions relative to that of the parent sample. DLS profiles of the three fractions are shown in Fig. 3, along with excitation-emission matrix scanning maps for each fraction collected under ambient conditions. The DLS profiles for fractions 1, 2, and 3 showed broad spectral shapes, and average diameters were estimated to be $5.4 \mathrm{~nm}(\sigma=3.5 \mathrm{~nm}), 4.0 \mathrm{~nm}(\sigma=2.8 \mathrm{~nm})$, and $2.9 \mathrm{~nm}(\sigma=2.1 \mathrm{~nm})$, respectively, indicating the reasonable order of elution on a size exclusion chromatography column. Optical absorption spectra of the three fractions are shown in Fig. 2(b). As expected, the blueshift of absorption edge with decreasing average size of ncGe was observed. The excitation-emission matrix scanning was performed for each fraction, and the maps are displayed in Fig. 3. The spectra for the fractions 1, 2, and 3 showed significantly broad emission profiles, each centered on emission wavelengths of approximately 480, 380, and $340 \mathrm{~nm}$, respectively. These broad spectral line shapes are caused by the polydispersed ncGe for each fraction. A $2.9 \mathrm{~nm}$ of ncGe shows PL spectrum with an emission maximum at around $340 \mathrm{~nm}$. 
Obviously, PL spectra shift to long-wavelength side with increase in physical-size of ncGe. Major components of visible PL features were isolated as the fraction 1. It is considered that the fraction 1 is composed of several species having different emission spectroscopic characteristics. In fact, the emission maxima of PL spectra were dependent of the excitation wavelength even in the same sample. The chromaticity diagram of the fraction 1 is shown in Fig. 4. Although these white lights have poorer color rendering index (CRI) owing to the unbalanced red-emission color, this result is of interest since currently the color of white light is tuned with excitation as marked. In our previous paper, the parent sample prepared by laser chemical synthesis was subjected to color separation with conventional silica-gel column chromatography followed by HPLC to separate the ncGe into UV-, violet-, blue-, light-blue, and green-emitting fractions, respectively [19]. However, we cannot obtain ncGe emitting the lights in the longer-visible regions such as yellow and red using the laser ablation method because of the rapid reaction between ncGe with surface radicals and surrounding 1-alkens to yield small size of ncGe. However, the present method provides much thermal energy enough for growing nanocrystals bigger than $5 \mathrm{~nm}$, and offers a reaction field in which the growing ncGe are slowly terminated with surrounding molecules compared to the field produced by laser ablation. As a result, the colloidal sample including the yellow-, orange-, and red-emitting ncGe which are indispensable color components for producing a white light was obtained unlike the ncGe sample prepared by 
laser chemical synthesis. Addition of red-emission color may lead to the full-control over white

light emission in the wide ranging from warm-white to cool-white.

\section{Conclusions}

This paper presented the first solution-phase synthesis of ncGe emitting the visible lights by heating $\mathrm{GeI}_{2}$ in oleylamine. The reaction at $300^{\circ} \mathrm{C}$ offered the opportunity to form a diamond cubic Ge lattice. The excitation spectroscopic study concluded that the broad PL spectrum was described as ensemble of different emission spectral lines. Therefore, the extraction of the specific nanocrystals with predefined emission property allowed for the tuning the color of white PL spectrum. The extraction of ncGe was performed using conventional silica-gel column chromatography and subsequent HPLC with size exclusion column. This paper emphasizes the efficiency of "separation of nanoparticles by emission" to create the predefined emission spectrum.

\section{Acknowledgements}

The use of facilities in Nanotechnology Innovation Center in NIMS is highly appreciated. This work was, in part, supported by JST PRESTO, the Grant-in-Aid for challenging Exploratory Research, MEXT, and Konica Minolta Imaging Science Award, 
Konica Minolta Science and Technology Foundation, Japan.

\section{References}

[1] Q. Dai, C. E. Duty, M. Z. Hu, Small 6 (20109 1577.

[2] J. Zhou, N. Shirahata, H. T. Sun, B. Ghosh, M. Ogawara, Y. Teng, S. Zhou, S. C. R. Gui, M. Fujii, J. Qiu, J. Phys. Chem. Lett. 4 (2013) 402.

[3] B. C. Cheng, Y. H. Xiao, G. S.Wu, L. D. Zhang, Adv. Funt. Mater. 14 (2004) 913.

[4] W. Di, X. Ren, N. Shirahata, C. Liu, L. Zhang, Y. Sakka, N. Pinna, CrystEngComm 13 (2011) 5226.

[5] L. Li, C. K. Tsung, Z. Yang, G. D. Stucky, L. D. Sun, J. F. Wang, C. H. Yan, Adv. Mater. 20 (2008) 903.

[6] H. T. Sun, Y. Matsushita, Y. Sakka, N. Shirahata, M. Tanaka, Y. Katsuya, H. Gao, K. Kobayashi, J. Am. Chem. Soc. 134 (2012) 2918

[7] T. Erdem, H. V. Demir, Nature Photon. 5 (2011) 126.

[8] S. S. Babu, J. Aimi, H. Ozawa, N. Shirahata, A. Saeki, S. Seki, A. Ajayaghosh, H. Möhwald, T. Nakanishi, Angew. Chem. Int. Ed. 51 (2012) 3391.

[9] T. J. Pennycook, J. R. McBride, S. J. Rosenthal, S. J. Pennycook, S. T. Pantelides, Nano Lett. 12 (2012) 3038 .

[10] M. M. Krause, J. Mooney, P. Kambhampati, ACS Nano 7 (2013) 5922.

[11] S. Kim, T. Kim, M. Kang, S. K. Kwak, T. W. Yoo, L. S. Park, I. Yang, S. Hwang, J. E. Lee, S. K. Kim, S. -W. Kim, J. Am. Chem. Soc. 134 (2012) 3804.

[12] B. Ghosh, M. Ogawara, Y. Sakka, N. Shirahata, Chem. Lett. 41 (2012) 1157.

[13] Y. Maeda, N. Tsukamoto, Y. Yazawa, Y. Kanemitsu, Y. Masumoto, Appl. Phys. Lett., 59, 24, $1991,3168$.

[14] X. Lu, K. J. Ziegler, A. Ghezelbash, K. P. Johnston, B. A. Korgel, Nano Lett. 4 (2004) 969.

[15] B. Ghosh, Y. Sakka, N. Shirahata, J. Mater. Chem. A 1 (2013) 3747.

[16] B. R. Taylor, S. M. Kauzlarich, H. W. H. Lee, G. R. Delgado, Chem. Mater. 10 (1998) 22.

[17] D. C. Lee, J. M. Pietryga, I. Robel, D. J. Werder, R. D. Schaller, V. I. Klimov, J. Am. Chem. Soc. 131 (2009) 3436.

[18] E. J. Henderson, C. M. Hessel, J. G. C. Veinot, J. Am. Chem. Soc. 130 (2008) 3624.

[19] N. Shirahata, D. Hirakawa, Y. Masuda, Y. Sakka, Langmuir 29 (2013) 7401.

[20] E. J. Henderson, M. Seino, D. P. Puzzo, G. A. Ozin, ACS Nano 4 (2010) 7683.

[21] Z. C. Holman, U. R. Kortshagen, Nano Lett. 11 (2011) 2013. 
[22] N. H. Chou, K. D. Oyler, N. E. Motl, R. E. Schaak, Chem. Mater. 21 (2009) 4105.

[23] B. Ghosh, M. Ogawara, Y. Sakka, N. Shirahata, J. Nanosci. Nanotechnol. 13 (2013) in press

[24] D. A. Ruddy, J. C. Johnson, E. R. Smith, N. R. Neale, ACS Nano 4 (2010) 7459.

[25] X. Lu, B. A. Korgel, K. P. Johnston, Chem. Mater. 17 (2005) 6479.

[26] D. C. Cronemeyer, J. Appl. Phys. 29 (1958) 1730.

[27] M. Fujii, S. Hayashi, K. Yamamoto, Appl. Phys. Lett. 57 (1990) 2692.

[28] N. Shirahata, M. R. Linford, S. Furumi, L. Pei, Y. Sakka, R. G. Gates, M. C. Aspland, Chem. Commun. (2009) 4684.

[29] S. Wei, T. Yamamura, D. Kajiya, K. Saitow, J. Phys. Chem. C 116 (2012) 3928.

[30] S. Lee, W. J. Cho, I. K. Han, W. J. Choi, J. I. Lee, Phys. Stat. Soli. B 241 (2004) 2767.

[31] N. Shirahata, D. Hirakawa, Y. Sakka, Green Chem. 12 (2010) 2139.

[32] J. C. Vinci, L. A. Colon, Anal. Chem. 84, 1178.

[33] M. Sakamoto, D. Tanaka, H. Tsunoyama, T. Tsukuda, Y. Minagawa, Y. Majima, T. Teranishi, J. Am. Chem. Soc. 134 (2012) 816.

[34] A. Henglein, J. Phys. Chem. 97 (1993) 5457. 


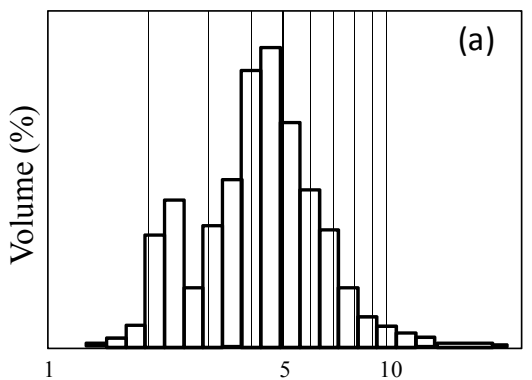

Diameter (nm)
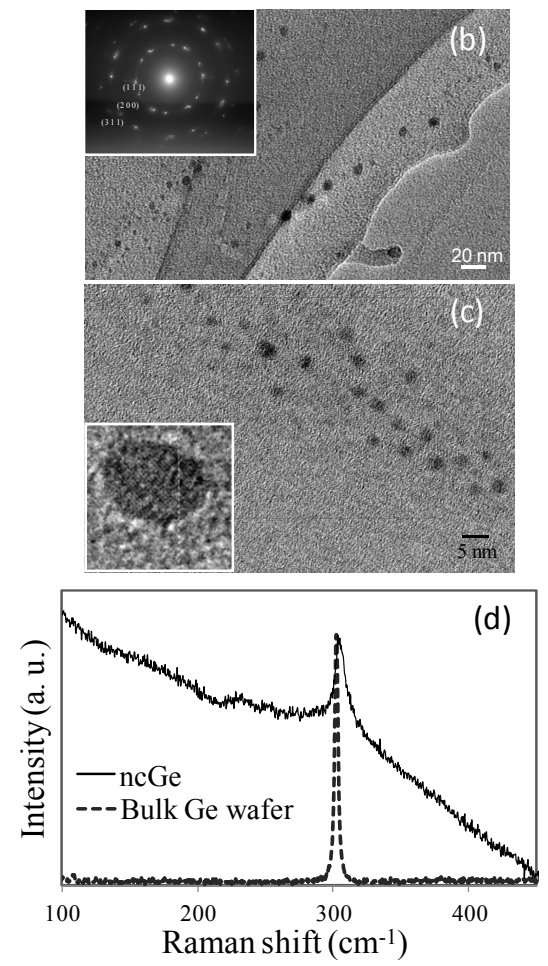

Fig. 1. (a) DLS profile, (b) TEM photograph with SADP, (c) high-resolution TEM image, and (d) Raman spectrum of the parent ncGe sample. 

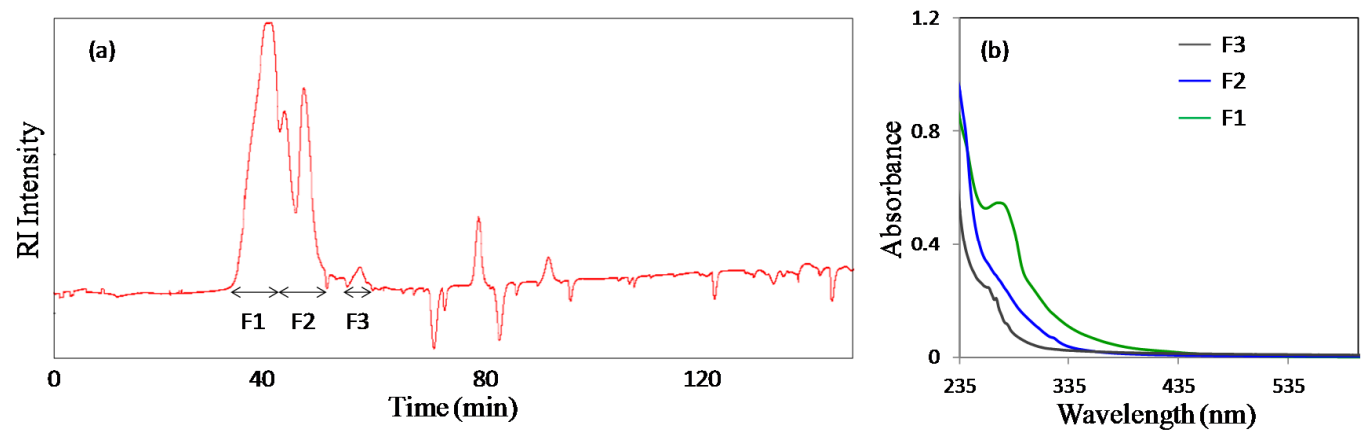

Fig. 3. (a) HPLC chromatogram with RI detection and (b) UV-VIS spectra of the three HPLC-derived fractions

Fig. 2. (a) HPLC chromatogram with RI detection and (b) UV-VIS spectra of the three HPLC-derived fractions
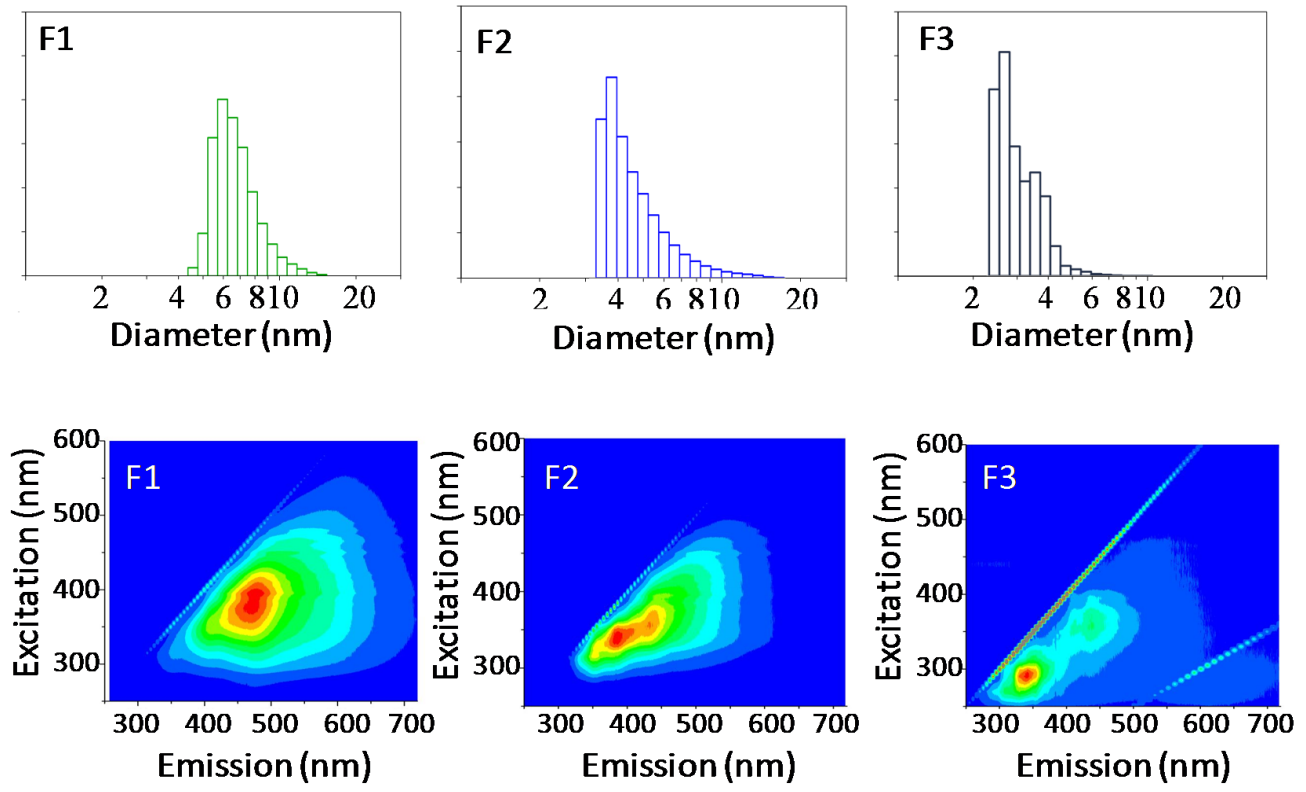

Fig. 3. DLS profiles and Excitation-Emission scanning map for the three HPLC-derived fractions. The average sizes $\left(d_{\mathrm{avr}}\right)$ and their standard deviations $(\sigma)$ for each fraction are estimated to be $\left(d_{\mathrm{avr}}\right.$, $\sigma)=\mathrm{F} 1(5.4 \mathrm{~nm}, 3.5 \mathrm{~nm}), \mathrm{F} 2(4.0 \mathrm{~nm}, 2.8 \mathrm{~nm})$, and F3 (2.9 nm, $2.1 \mathrm{~nm})$, respectively. 


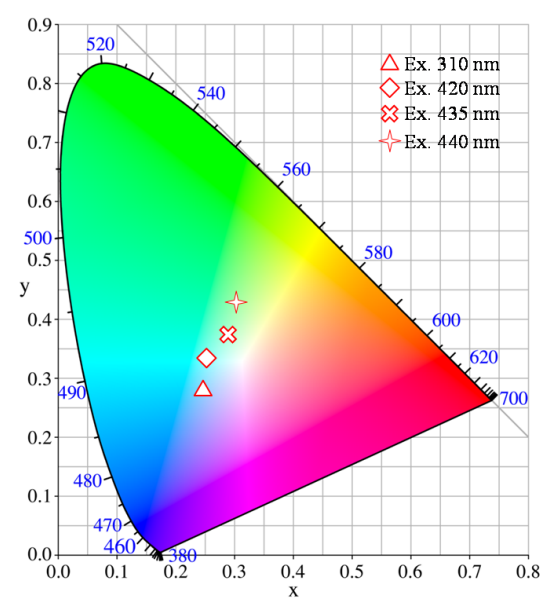

Fig. 4. CIE coordinates of the $5.4 \mathrm{~nm}-\mathrm{ncGe}$ at different excitations.

\section{Graphical Abstract}

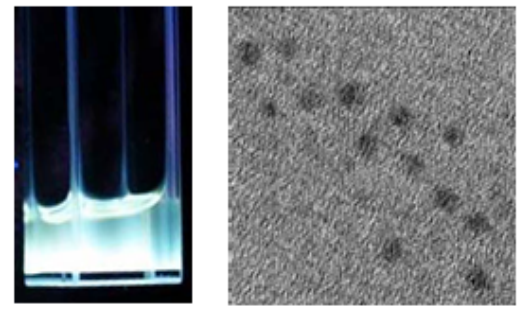

